\title{
Hydrothermal Decarboxylation and Hydrogenation of Fatty Acids over $\mathrm{Pt} / \mathrm{C}$
}

\author{
Jie $\mathrm{Fu}_{1}^{[a, b]}$ Xiuyang $\mathrm{Lu}_{1}^{[\mathrm{b}]}$ and Phillip E. Savage ${ }^{*[a]}$
}

We report herein on the conversion of saturated and unsaturated fatty acids to alkanes over $\mathrm{Pt} / \mathrm{C}$ in high-temperature water. The reactions were done with no added $\mathrm{H}_{2}$. The saturated fatty acids (stearic, palmitic, and lauric acid) gave the corresponding decarboxylation products ( $n$-alkanes) with greater than $90 \%$ selectivity, and the formation rates were independent of the fatty acid carbon number. The unsaturated fatty acids (oleic and linoleic acid) exhibited low selectivities to the decarboxylation product. Rather, the main pathway was hydrogenation to from stearic acid, the corresponding saturated fatty acid. This compound then underwent decarboxylation to form heptadecane. On the basis of these results, it appears that this reaction system promotes in situ $\mathrm{H}_{2}$ formation. This hydrothermal decarboxylation route represents a new path for using renewable resources to make molecules with value as liquid transportation fuels.

\section{Introduction}

Natural oils and fats (e.g., plant and seed oils) are complex mixtures of triglycerides, which consist of a glycerol backbone with three fatty acid moieties. The fatty acids can be either saturated or unsaturated, and the carbon numbers range from about $\mathrm{C} 8$ to $\mathrm{C} 24$, but $\mathrm{C} 12, \mathrm{C} 16$, and $\mathrm{C} 18$ fatty acids tend to be the most abundant. These natural materials can serve as feedstocks for the production of renewable liquid transportation fuels, such as biodiesel (e.g., fatty acid methyl esters) or green diesel (e.g., hydrocarbons). There is considerable interest in producing hydrocarbons from the fatty acid moieties in triglycerides because hydrocarbon molecules would, in principle, be compatible with the existing distribution infrastructure developed for petroleum-derived liquid transportation fuels.

Recent review articles have highlighted the significant research and development (R\&D) efforts that have been devoted to using and adapting petroleum hydrotreatment technology to convert triglycerides and fatty acids into hydrocarbons. ${ }^{[1,2]}$ These efforts typically involve conventional hydrotreatment catalysts (e.g., CoMo/ $/ \mathrm{Al}_{2} \mathrm{O}_{3} \mathrm{NiMo} / \mathrm{Al}_{2} \mathrm{O}_{3}$ ) and reaction conditions. A high $\mathrm{H}_{2}$ partial pressure is required to remove the oxygen atoms in the fatty acids as $\mathrm{H}_{2} \mathrm{O}$ molecules. UOP/Eni and Neste Oil have developed hydrotreatment processes for vegetable and seed oils that can operate on a large scale. The high $\mathrm{H}_{2}$ consumption associated with these processes is their main drawback. $\mathrm{H}_{2}$ is not currently available in large quantities from renewable resources and $\mathrm{H}_{2}$ costs can be high. Moreover, $\mathrm{H}_{2}$ is made primarily from steam reforming of natural gas and $\mathrm{CO}_{2}$ is the byproduct. Thus, a near-term process for the production of fully renewable biofuels needs to operate without added $\mathrm{H}_{2}$.

Recognizing the advantages of both a hydrocarbon biofuel and reducing the amount of $\mathrm{H}_{2}$ needed to produce it from plant oils, several labs have been exploring decarboxylation chemistry as an alternative to hydrodeoxygenation. ${ }^{[3-17]}$ Decarboxylation removes the $\mathrm{O}$ atoms in fatty acids as $\mathrm{CO}_{2}$ rather than $\mathrm{H}_{2} \mathrm{O}$. Thus, stoichiometrically no $\mathrm{H}_{2}$ is required for the reaction. Moreover, the loss of a $\mathrm{CO}_{2}$ molecule does not lead to a loss in any of the chemical energy in the molecule. In fact, it produces a hydrocarbon molecule with an even higher energy density. This path is an attractive one for making renewable hydrocarbon fuels, but aside from our recent report ${ }^{[3]}$ and a set of earlier catalyst screening experiments, ${ }^{[18]}$ all previous work on fatty acid decarboxylation has been done in organic reaction media, such as dodecane or mesitylene. In contrast, the focus herein is on fatty acid decarboxylation in water. This hydrothermal decarboxylation is technologically important, but the reaction pathways are essentially unexplored.

There are many biofuel production schemes in which fatty acids are produced in an aqueous phase. One example is Kusdiana and Saka's two-step process for handling low-cost triglyceride-containing feedstocks. ${ }^{[19]}$ The first step is hydrolysis of the triglycerides to produce an aqueous stream of fatty acids. They proposed to recover the fatty acids and then convert them to biodiesel, but one could also, and perhaps even more simply, perform a hydrothermal catalytic decarboxylation to produce alkanes. This alternate second step would eliminate the need to separate the fatty acids from water and it would produce a hydrocarbon biofuel. Another aqueous phase process is that of Centia for making biofuel from triglycerides. ${ }^{[20]}$ This process, now in scaleup by Diversified Energy, uses a hy-

[a] Dr. J. Fu, Prof. P. E. Savage

Department of Chemical Engineering

University of Michigan

Ann Arbor, MI, 48109 (USA)

Fax: (+ 1) (734) 763-0459

E-mail:psavage@umich.edu

[b] Dr. J. Fu, Prof. X. Lu

Department of Chemical and Biological Engineering

Zhejiang University

Hangzhou 310027 (PR China) 
drolysis step to convert triglycerides into free fatty acids. Again the fatty acids are separated from the water and then decarboxylated in an organic solvent. The process can probably be simplified by omitting the separation step and performing the decarboxylation in water. There are also recent reports on different hydrothermal processes for converting the lipids in wet algal biomass to either fatty solids ${ }^{[21]}$ or crude bio-oils with very high fatty acid contents. ${ }^{[22,23]}$ Rather than separating the water from the solids or bio-crudes, perhaps hydrothermal decarboxylation could be employed to produce a hydrocarbon fuel. Finally, Li et al. ${ }^{[24]}$ reported a catalytic hydrothermal conversion process for triglycerides, but again, they separated the fatty acids produced by hydrolysis from the aqueous phase and processed them in an organic solvent instead. It is clear that there are many potential biofuel processes under development in which aqueous streams of fatty acids are produced.

Our previous article showed that $\mathrm{Pt} / \mathrm{C}$ and $\mathrm{Pd} / \mathrm{C}$ catalysts are active for decarboxylation of a saturated fatty acid in an aqueous medium, with $\mathrm{Pt} / \mathrm{C}$ showing higher activity. ${ }^{[3]}$ Moreover, the work showed that no added $\mathrm{H}_{2}$ was required to do the decarboxylation. Herein, we provide more detailed information about the hydrothermal reaction pathways and kinetics for this catalyzed transformation.

Watanabe and co-workers provide the only other report on hydrothermal decarboxylation of fatty acids. ${ }^{[18]}$ Some of the materials they tested (e.g., $\mathrm{NaOH}, \mathrm{KOH}$ ) on stearic acid in supercritical water at $400^{\circ} \mathrm{C}$ produced the decarboxylation product $\left(\mathrm{C}_{17}\right.$ alkane). Other materials (e.g., $\left.\mathrm{CeO}_{2}, \mathrm{Y}_{2} \mathrm{O}_{3}, \mathrm{ZrO}_{2}\right)$ produced the $C_{16}$ alkene. None of the materials they tested were as active as $\mathrm{Pt} / \mathrm{C}$.

Herein, we report on the catalytic hydrothermal decarboxylation of five different fatty acids that are prevalent in nature: stearic (18:0), palmitic (16:0), lauric (12:0), oleic (18:1), and linoleic (18:2) acids. The numbers in parentheses indicate, respectively, the number of carbon atoms and number of double bonds in each of the fatty acids. This suite of compounds allowed us to determine the influence of the fatty acid carbon chain length and its degree of unsaturation on the Pt/C-catalyzed hydrothermal decarboxylation of fatty acids.

\section{Experimental Section}

We conducted reactions in unstirred mini-batch reactors assembled from 3/8 inch stainless steel Swagelok parts. Port connectors sealed with a cap on each end gave a reactor volume of about $1.67 \mathrm{~mL}$. Prior to use in any experiments, reactors were rinsed with acetone, dried, loaded with water $(1.1 \mathrm{~mL})$, and conditioned at $300^{\circ} \mathrm{C}$ for $30 \mathrm{~min}$ to remove any residual materials remaining from the manufacture of the metal parts. Stearic, palmitic, lauric, oleic, and linoleic acids; heptadecane; undecane; and $5 \% \mathrm{Pt} / \mathrm{C}$ catalyst were purchased from Sigma-Aldrich. Pentadecane was purchased from Wiley. We have previously reported details about the catalyst activity maintenance, surface area, pore size, and metal dispersion. ${ }^{[3]}$ Briefly, the catalyst particle diameter was $50 \mu \mathrm{m}$, the surface area of the fresh catalyst was $1483 \mathrm{~m}^{2} \mathrm{~g}^{-1}$, and the metal dispersion was $39 \%$. HPLC grade acetone was obtained from Fisher Scientific. All chemicals were used as received.
Unless otherwise indicated, each experiment was conducted at $330^{\circ} \mathrm{C}$ with $0.176 \mathrm{mmol}$ of fatty acid and $5 \mathrm{mg} \mathrm{Pt} / \mathrm{C}$ in each reactor. The amount of water loaded was such that the expanded liquid aqueous phase occupied $95 \%$ of the reactor volume under the reaction conditions, based on the saturation densities ${ }^{[25]}$ of pure water at the reaction temperatures. After being loaded, each reactor was sealed by attaching and then tightening the reactor cap. The reactor headspace contained air, but the amount of residual $\mathrm{O}_{2}$ present was enough to oxidize completely no more than $0.2 \%$ (by moles) of the fatty acid. No $\mathrm{H}_{2}$ was added to the reactors in any of these experiments.

Sealed reactors containing fatty acid, $\mathrm{Pt} / \mathrm{C}$, and water were placed in a Techne SBL-2 fluidized sand bath preheated to the desired reaction temperature, controlled to within $1{ }^{\circ} \mathrm{C}$. The time required for these reactors to reach reaction temperature was roughly $2 \mathrm{~min}$. After the desired reaction time was completed, the reactors were removed from the sand bath and submerged in a water bath at ambient temperature to quench the reaction. The reactor contents were transferred to a volumetric flask, and the reactors were subsequently rinsed with repeated acetone washes until the total volume collected was $10 \mathrm{~mL}$.

The reaction products were identified by fragmentation patterns from an Agilent 5970 mass spectrometric detector and by matching gas chromatograph retention times with known standards. Product separation for saturated fatty acids was achieved by using an Agilent 6890 gas chromatograph equipped with a $50 \mathrm{~m} \times$ $0.2 \mathrm{~mm} \times 0.33 \mu \mathrm{m}$ capillary column (HP-5 for flame ionization detection (FID) and HP-5MS for MS detection). The injection port temperature was $325^{\circ} \mathrm{C}$ and the temperature program consisted of a 5 min soak at $40^{\circ} \mathrm{C}$ followed by a $10^{\circ} \mathrm{Cmin}^{-1}$ ramp up to a final temperature of $290^{\circ} \mathrm{C}$, which was then held for $15 \mathrm{~min}$. For the unsaturated fatty acids, product separation was achieved by using an Agilent $6890 \mathrm{GC}$ equipped with a $30 \mathrm{~m} \times 0.32 \mathrm{~mm} \times 0.25 \mu \mathrm{m}$ Nukol capillary column. The injection port temperature was $220^{\circ} \mathrm{C}$ and the temperature program consisted of a $2 \mathrm{~min}$ soak at $60^{\circ} \mathrm{C}$ followed by a $10^{\circ} \mathrm{Cmin}^{-1}$ ramp up to a final temperature of $200^{\circ} \mathrm{C}$, which was then held for $55 \mathrm{~min}$.

Quantitative analysis was accomplished by generating and using calibration curves for each compound of interest. The FID response was linear for each component over the concentration ranges used. Minor products for which we did not have authentic standards were quantified by assuming their detector responses were similar to those of a compound that eluted with a similar retention time and for which we had an experimental calibration curve.

Product yields were calculated as the amount (moles or mass) of product recovered divided by the initial amount (moles or mass) of fatty acid loaded into the reactor. Selectivities were calculated as the number of moles of product recovered divided by the number of moles of fatty acid that had reacted (i.e., molar yield/conversion). Uncertainties reported herein are standard deviations, which were determined by replicating the experiments. Each data point represents the mean result from at least three independent experiments.

\section{Results and Discussion}

This section presents and interprets the experimental results and places them in the context of previous work done on the catalytic decarboxylation of both saturated and unsaturated fatty acids over Pt/C. Our earlier analysis used the Weisz-Prater criterion to show that the experimental system we used can provide access to intrinsic reactivities unfettered by mass trans- 
port limitations. ${ }^{[3]}$ Moreover, our previous work showed that the catalyst can be reused at least three times with no appreciable loss in activity.

\section{Decarboxylation of saturated fatty acids}

Figures 1 and 2 show the temporal variation of the molar yield of and the selectivity to the $C_{N-1}$ alkane that arises from the decarboxylation of the $C_{N}$ saturated fatty acids at $330^{\circ} \mathrm{C}$. The

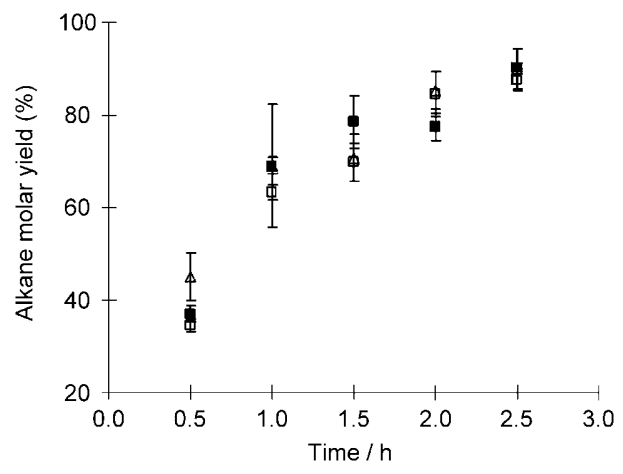

Figure 1. Temporal variation of alkane molar yields from decarboxylation of stearic acid $(\mathbf{\square})$, palmitic acid $(\square)$, and lauric acid $(\triangle)$.

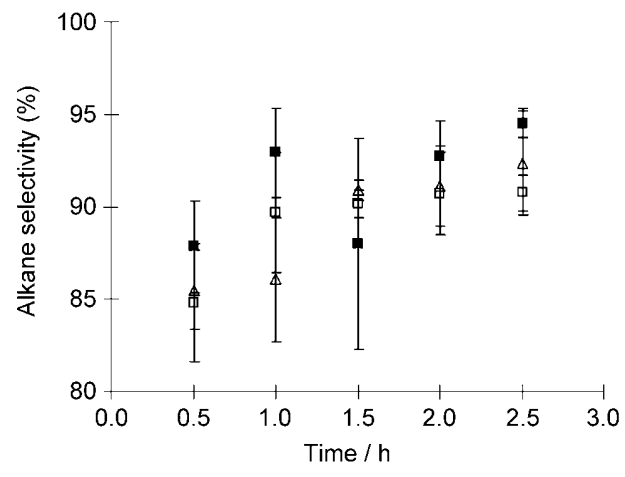

Figure 2. Temporal variation of alkane selectivities from decarboxylation of stearic acid (घ), palmitic acid ( $\square)$, and lauric acid $(\triangle)$.

data for palmitic acid are from our earlier work, ${ }^{[3]}$ wherein we used a $0.195 \mathrm{mmol}$ reactant loading. The decarboxylation rates and selectivities to alkanes are very similar for the three saturated fatty acids, which suggest that the decarboxylation rates are independent of the carbon number for the saturated fatty acids we tested. Additionally, the high selectivities (85-95\%) indicated in Figure 2 show that the saturated fatty acids can be selectively decarboxylated over $\mathrm{Pt} / \mathrm{C}$ in high-temperature water. The selectivities were not $100 \%$, however, which indicates that other products were also formed, albeit in much lower yields. These minor products were primarily $C_{7}$ to $C_{N} n$-alkanes.

These results extend our previous work by showing that the C18 fatty acid (stearic) and the C12 fatty acid (lauric) behave similarly to the $\mathrm{C} 16$ acid (palmitic) that we had reported previously. ${ }^{[3]}$ It was expected that the chain length would not have a discernible influence on the decarboxylation rate. The catalytic chemistry occurs at the carboxylic acid end of the molecule and one would expect those catalyst-molecule interactions to be largely insensitive to the addition or removal of a few carbon atoms from the other end of the fatty acid molecule. Moreover, recent work on fatty acid decarboxylation over $\mathrm{Pd}$ catalysts in organic solvents has also demonstrated the insensitivity of the reaction rate to the fatty acid chain length. ${ }^{[7,17]}$

\section{Decarboxylation of unsaturated C18 fatty acids}

Having found that the decarboxylation of saturated fatty acids was insensitive to the fatty acid alkyl chain length (at least within the C12-C18 range), we next explored the influence of the degree of unsaturation on decarboxylation. Figures 3 and 4 show the temporal variation of the molar yield of and selec-

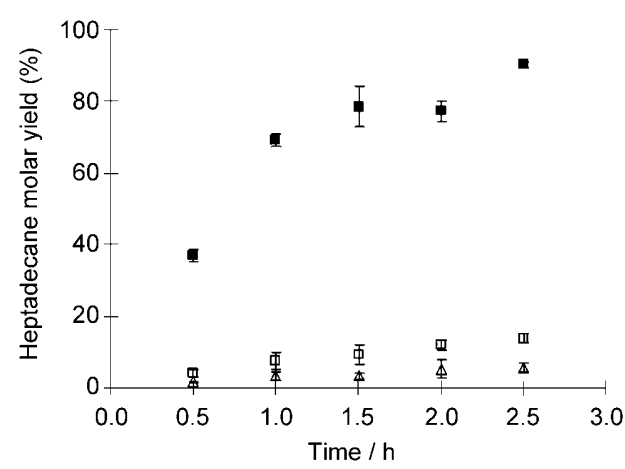

Figure 3. Temporal variation of heptadecane molar yields from stearic acid $(\boldsymbol{\square})$, oleic acid $(\square)$, and linoleic acid $(\triangle)$.

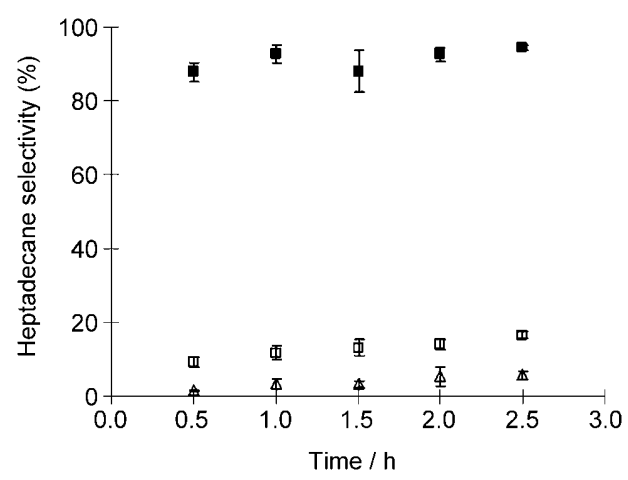

Figure 4. Temporal variation of heptadecane selectivity from stearic acid (घ), oleic acid $(\square)$, and linoleic acid $(\triangle)$.

tivity to heptadecane, the $C_{N-1}$ alkane that would form from the decarboxylation of a C18 fatty acid. Heptadecenes were also formed in the experiments with the unsaturated fatty acids, but their yields were always lower than that of heptadecane, so we show only the heptadecane data here. Discussion of the complete product distribution will be deferred until the next section. 
Figures 3 and 4 show that the degree of unsaturation has a strong effect on the decarboxylation of fatty acids because the yields and selectivities from oleic acid (18:1) and linoleic acid (18:2) to heptadecane are much lower than those from stearic acid (18:0). Again, note that for both oleic and linoleic acid, the yield of heptadecane, although low, exceeded the yields of heptadecenes at all reaction times investigated. Thus, for these unsaturated fatty acids, it appears that a reaction other than decarboxylation is the main reaction path. Recall that no $\mathrm{H}_{2}$ was added to the reactor. The next section explores the reaction pathways in more detail.

\section{Oleic acid reaction pathway}

Figure 5 shows the temporal variation of the molar yield of oleic acid and the major reaction products formed at $330^{\circ} \mathrm{C}$. Stearic acid, the product of oleic acid hydrogenation, is the

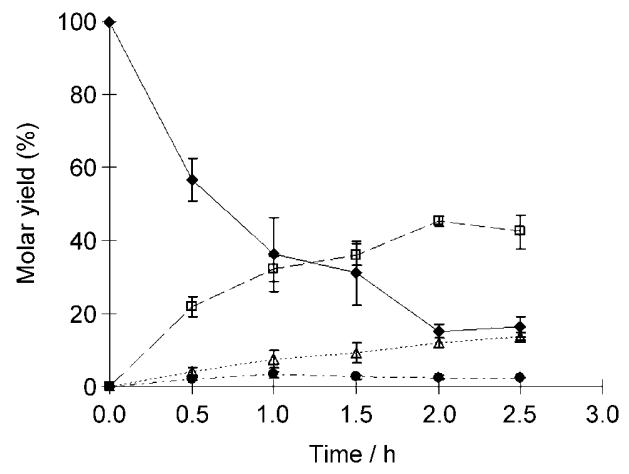

Figure 5. Temporal variation of product molar yields (stearic acid ( $\square$ ), heptadecane $(\triangle)$, and heptadecenes $(\bullet))$ from oleic acid $(\bullet)$.

major product at all times. The yield of stearic acid increases with time until about $2 \mathrm{~h}$ when it appears to plateau or perhaps even begin to slowly decrease. The molar yield of heptadecane increased steadily with reaction time, and it was always present in higher yields than the heptadecenes (e.g., 8-heptadecene, 3-heptadecene), which are the decarboxylation product(s) from oleic acid. The trends in Figure 5 suggest that stearic acid is the intermediate product between oleic acid and heptadecane. That is, oleic acid was hydrogenated first, and then the stearic acid produced was decarboxylated to heptadecane. A similar sequential hydrogenation-decarboxylation pathway for oleic acid was proposed by Immer et al. for the reaction in a dodecane solvent..$^{[9]}$

$\mathrm{Pt}$ is a good hydrogenation catalyst ${ }^{[26,27]}$ and to determine the role of this catalyst on the hydrogenation pathway we carried out experiments with oleic acid at $330^{\circ} \mathrm{C}$ for $1 \mathrm{~h}$, but without Pt/C. No heptadecane was detected, and the yield to stearic acid was $(4.0 \pm 1.7) \%$; this is much lower than the yield to stearic acid in the presence of $\mathrm{Pt} / \mathrm{C}$. Nevertheless, this result indicates some capacity for hydrogenation in high-temperature water alone, perhaps catalyzed by the stainless-steel reactor walls. Of course, hydrogen is required for hydrogenation to occur. Since no $\mathrm{H}_{2}$ was loaded into the reactor in any of these experiments, the $\mathrm{H}_{2}$ must be formed in situ during the reaction. To test this hypothesis of in situ $\mathrm{H}_{2}$ formation, we conducted an experiment wherein oleic acid $(0.195 \mathrm{mmol})$ was reacted over $\mathrm{Pt} / \mathrm{C}(10 \mathrm{mg})$ at $370^{\circ} \mathrm{C}$ for $3 \mathrm{~h}$ in a reactor equipped with a valve so that we could collect and analyze the gasphase reaction products. This experiment revealed a $30 \% \mathrm{H}_{2}$ molar yield, so $\mathrm{H}_{2}$ is indeed formed in situ during the reaction. $\mathrm{CO}_{2}, \mathrm{CH}_{4}$, and $\mathrm{C}_{2} \mathrm{H}_{6}$ were also detected, with molar yields of 100,10 , and $5.1 \%$, respectively. Pt catalysts are commonly used for aqueous-phase reforming of biomass-derived molecules, ${ }^{[28,29]}$ and the gas products we detected are typically observed during aqueous-phase reforming. ${ }^{[30]}$ Therefore, the Pt/C catalyst in our system might be playing this role by converting a small amount of the oleic acid to $\mathrm{H}_{2}$. Of course, a variety of reactions such as $\mathrm{C}-\mathrm{C}$ bond cleavage and water-gas shift occur during aqueous-phase reforming to generate $\mathrm{H}_{2}$ molecules. The rate and selectivity for $\mathrm{H}_{2}$ production is a function of both the metal catalyst and the support.

Table 1 shows a more complete itemization of the products identified from the hydrothermal reaction of oleic acid over Pt/ C. In addition to the major products illustrated in Figure 5, the

\begin{tabular}{|ll|}
\hline Table 1. Products from oleic acid reactions over Pt/C at $330^{\circ} \mathrm{C}$ for $1.5 \mathrm{~h}$ \\
Compound & Yield [wt \%] \\
\hline 2-heptanone & $0.54 \pm 0.02$ \\
2-pentanone-4-hydroxy-4-methyl & $0.08 \pm 0.01$ \\
tetradecane & $0.05 \pm 0.01$ \\
pentadecane & $0.10 \pm 0.06$ \\
hexadecane & $0.16 \pm 0.02$ \\
aromatic derivatives & $1.3 \pm 0.5$ \\
9-octadecanone & $0.15 \pm 0.17$ \\
1-propene-1-methoxy-2-methyl & $0.08 \pm 0.02$ \\
3-heptadecanone & $0.16 \pm 0.11$ \\
2-heptadecanone & $0.06 \pm 0.01$ \\
2(3 $H$ )-furanone-dihydro-5-tetradecyl & $3.9 \pm 0.1$ \\
heptadecane & $9.2 \pm 2.8$ \\
heptadecenes & $2.6 \pm 0.5$ \\
stearic acid & $36.1 \pm 2.9$ \\
oleic acid & $31.1 \pm 8.8$ \\
other & $7.0 \pm 1.2$ \\
\hline
\end{tabular}

reaction also produced lesser amounts of other products. The most abundant minor product was a tetradecylfuranone. The minor products also included ketones; $C_{14}, C_{15}$, and $C_{16}$ alkanes; and some aromatic compounds that could not be positively identified. We believe these are aromatic compounds because each contained a strong signal in the mass spectrum at $\mathrm{m} / \mathrm{z}$ 91, which is indicative of a tropylium ion $\left(\mathrm{C}_{7} \mathrm{H}_{7}^{+}\right)$; a signature signal for alkyl-substituted aromatic compounds. Likewise, the products we placed in the "other" category of Table 1 are ones that we were unable to identify. The product amounts in Table 1 sum to $93 \mathrm{wt} \%$, which is typical of the mass balances we routinely observed in the oleic acid experiments. Note that this overall mass balance does not statistically differ from $100 \%$ when one considers the uncertainty in the yields of the individual products. 
Taken collectively, the results in Figure 5 and Table 1 suggest that aqueous-phase reforming, hydrogenation, and decarboxylation are the main reaction paths for oleic acid over $\mathrm{Pt} / \mathrm{C}$. These combine to convert unsaturated fatty acids to alkanes. Along with these three reactions, other reactions such as cracking, aromatization, and cyclization occurred over $\mathrm{Pt} / \mathrm{C}$ in this hydrothermal medium.

\section{Linoleic acid reaction pathway}

Figure 6 shows the temporal variation of the product yields from hydrothermal treatment of linoleic acid at $330^{\circ} \mathrm{C}$ over the $5 \% \mathrm{Pt} / \mathrm{C}$ catalyst. The conversion of linoleic acid was $97 \%$ even
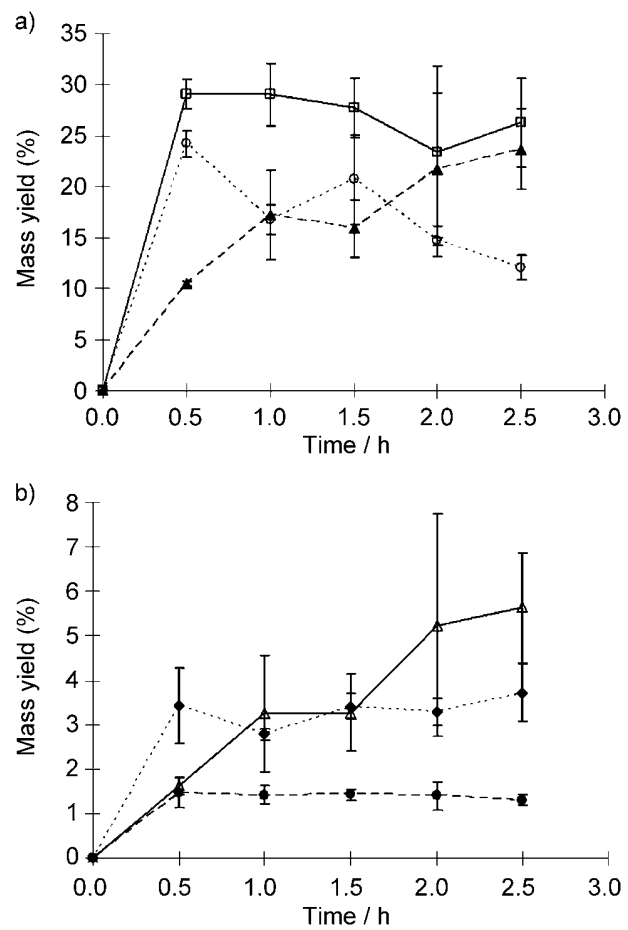

Figure 6. a) Temporal variation of mass yields of major products (oleic acid $(\square)$, stearic acid $(\mathbf{\Lambda})$, and heavy products $(\circ))$ from linoleic acid. b) Temporal variation of mass yields of minor products (heptadecane $(\triangle)$, heptadecenes $(\bullet)$, and light products $(\bullet))$ from linoleic acid.

at the shortest time investigated, so we did not include the low yields of remaining linoleic acid in Figure 6 . The disappearance of this polyunsaturated fatty acid was much more rapid than that of the monounsaturated oleic acid. The product spectrum from this polyunsaturated compound was also more complex. There were several products that we could not identify with confidence, even with the assistance of GC-MS analysis. Snåre et al. also observed a complicated product spectrum when working with linoleic acid. ${ }^{[8]}$ We lumped these products together as either "heavy" products, which elute from the GC near the linoleic acid reactant, or "light" products, which elute near heptadecane. We used the experimentally determined GC calibrations for oleic acid and heptadecane to quantify the mass yields of heavy and light products, respectively, that we could not positively identify. In doing so, we made the reasonable assumption that the FID response was proportional to the mass of carbon atoms in a given compound. Since we do not have identities for these heavy and light compounds and thus lack accurate molecular weights, we report the product yields on a mass rather than molar basis.

Figure 6 a shows that oleic acid was always the most abundant product. The heavy products were second in abundance at reaction times up to $1.5 \mathrm{~h}$. The yield of stearic acid was also high and it increased with time to become the second most abundant product after $1.5 \mathrm{~h}$. The yield reached about $25 \%$ after $2.5 \mathrm{~h}$. Figure $6 \mathrm{~b}$ shows that the yield of heptadecane increased steadily throughout the reaction, reaching about $5 \%$ after $2.5 \mathrm{~h}$. The yield of the light products showed a similar trend, whereas the yield of heptadecenes was largely stable at about $1 \%$. Recall that the yield of heptadecenes from oleic acid showed essentially the same trend.

Oleic acid and heavy products being the most abundant products at short reaction times is consistent with linoleic acid reacting first to form oleic acid and heavy products. The oleic acid can then be hydrogenated to form stearic acid, and the stearic acid undergoes decarboxylation to form heptadecane. The presence of heptadecenes indicates that decarboxylation is also a pathway, albeit minor, for linoleic and oleic acids. Kitayama and co-workers found that many kinds of monoenoic acids, including those with different double-bond positions and different conformations (trans or cis), formed during linoleic acid hydrogenation over $\mathrm{Pt} / \mathrm{Al}_{2} \mathrm{O}_{3}{ }^{\left[{ }^{[27]}\right.}$ The heavy products we observed could well correspond to compounds of this type. Figure 7 provides a schematic depiction of the major overall

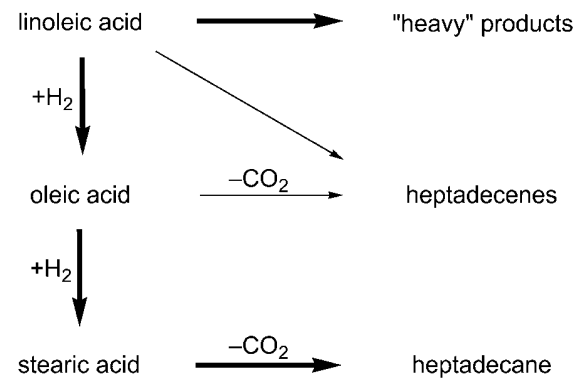

Figure 7. Hydrothermal catalytic reaction pathways for $\mathrm{C} 18$ fatty acids.

reaction pathways for hydrothermal decarboxylation of linoleic, oleic, and stearic acids. The thickness of each arrow in the reaction network is suggestive of the relative importance of that pathway. The pathways in Figure 7 are the same as those noted by Snåre et al. ${ }^{[8]}$ for linoleic acid decarboxylation in organic solvents, so the reaction medium does not appear to influence the main reaction pathways.

The mass balance for the linoleic acid experiments was about $75 \%$. We suspect that coupling reactions can occur with this polyunsaturated compound to produce higher-molecularweight material that would not elute from the GC and hence go undetected in our analysis. The literature lends some support for this hypothesis because Watanabe et al. observed cou- 
pling products in their study on fatty acids in supercritical water. $^{[18]}$ Snåre et al. also reported the formation of high-molecular-weight products from deoxygenation of unsaturated fatty acids. ${ }^{[8]}$

\section{Conclusion}

A commercial $5 \% \mathrm{Pt} / \mathrm{C}$ catalyst is active in hot water for the decarboxylation of saturated fatty acids (stearic, palmitic, and lauric acid). The selectivity to the decarboxylation product is about $90 \%$. No added $\mathrm{H}_{2}$ is required for this reaction. The decarboxylation rates for these three saturated fatty acids are independent of their carbon numbers. The unsaturated C18 fatty acids underwent decarboxylation much more slowly, and hydrogenation was the main reaction pathway even though no $\mathrm{H}_{2}$ was added to the reactor. The saturated fatty acid thus formed then underwent decarboxylation. These results suggest that $\mathrm{H}_{2}$ is formed in situ during this hydrothermal catalytic process.

Several general features noted herein for $\mathrm{Pt} / \mathrm{C}$-catalyzed hydrothermal decarboxylation (e.g., rate independent of fatty acid chain length, in situ $\mathrm{H}_{2}$ formation, hydrogenation of unsaturated fatty acids precedes appreciable decarboxylation, high selectivity to the decarboxylation product) have also been noted previously for fatty acid decarboxylation in organic solvents. Thus, the identity or nature of the solvent does not appear to have a strong influence on these aspects. Other features of this reacting system, however, such as the catalyst activity, the deactivation rate, and the ease of in situ $\mathrm{H}_{2}$ formation may yet be sensitive to the reaction medium. Additional research is required to address these issues.

\section{Acknowledgements}

J.F. thanks the China Scholarship Council for financial support as a joint PhD student, and we acknowledge financial support from the U-M College of Engineering and the National Science Foundation (CBET-0755617, EFRI-0937992).

Keywords: biofuels · biomass - green diesel $\cdot$ heterogeneous catalysis $\cdot$ reaction pathways

[1] S. Lestari, P. Mäki-Arvela, J. Beltramini, G. Q. M. Lu, D. Y. Murzin, ChemSus Chem 2009, 2, 1109-1119.
[2] B. Smith, H. C. Greenwell, A. Whiting, Energy Environ. Sci. 2009, 2, 262 271.

[3] J. Fu, X. Lu, P. E. Savage, Energy Environ. Sci. 2010, 3, 311-317.

[4] P. Maki-Arvela, I. Kubickova, M. Snåre, K. Eranen, D. Y. Murzin, Energy Fuels 2007, 21, 30-41.

[5] J. G. Immer, H. H. Lamb, Energy Fuels 2010, 24, 5291-5299.

[6] S. Lestari, P. Maki-Arvela, K. Eranen, J. Beltramini, G. Q. Max Lu, D. Y. Murzin, Catal. Lett. 2010, 134, 250-257.

[7] S. Lestari, P. Maki-Arvela, I. Simakova, J. Beltramini, G. Q. Max Lu, D. Y. Murzin, Catal. Lett. 2009, 130, 48-51.

[8] M. Snåre, I. Kubičková, P. Mäki-Arvela, D. Chichova, K. Eränen, D. Yu. Murzin, Fuel 2008, 87, 933-945.

[9] J. G. Immer, M. J. Kelly, H. H. Lamb, Appl. Catal. A: Gen. 2010, 375, $134-$ 139.

[10] M. Snåre, I. Kubičková, P. Mäki-Arvela, K. Eränen, D. Y. Murzin, Ind. Eng. Chem. Res. 2006, 45, 5708-5715.

[11] H. Bernas, K. Eränen, I. Simakova, A-R Leino, K. Kordás, J. Myllyoja, P. Mäki-Arvela, T. Salmi, D. Y. Murzin, Fuel 2010, 89, 2033-2039.

[12] I. Simakova, O. Simakova, P. Mäki-Arvela, A. Simakov, M. Estrada, D. Y. Murzin, Appl. Catal. A: Gen. 2009, 355, 100-108.

[13] S. Lestari, I. Simakova, A. Tokarev, P. Mäki-Arvela, K. Eränen, D. Y. Murzin, Catal. Lett. 2008, 122, 247-251.

[14] E. W. Ping, R. Wallace, J. Pierson, T. F. Fuller, C. W. Jones, Microporous Mesoporous Mater. 2010, 132, 174-180.

[15] P. T. Do, M. Chiappero, L. L. Lobban, D. E. Resasco, Catal. Lett. 2009, 130, 9-18.

[16] B. Rozmysłowicz, P. Mäki-Arvela, S. Lestari, O. A. Simakova, K. Eränen, I. L. Simakova, D. Y. Murzin, T. O. Salmi, Top. Catal. 2010, 53, 1274-1277.

[17] I. Simakova, O. Simakova, P. Mäki-Arvela, D. Y. Murzin, Catal. Today 2010, $150,28-31$

[18] M. Watanabe, T. lida, H. Inomata, Energy Convers. Manage. 2006, 47, $3344-3350$.

[19] D. Kusdiana, S. Saka, Appl. Biochem. Biotechnol. 2004, 115, 0781-0791.

[20] http://www.diversified-energy.com/index.cfm?s_webAction = centia, accessed October 2010.

[21] R. B. Levine, T. Pinnarat, P. E. Savage, Energy Fuels 2010, 24, 5235-5243.

[22] T. Brown, P. Duan, P. E. Savage, Energy Fuels 2010, 24, 3639-3646.

[23] P. Duan, P. E. Savage, Ind. Eng. Chem. Res. 2011, 50, 52-61.

[24] L. Li, E. Coppola, J. Rine, J. L. Miller, D. Walker, Energy Fuels 2010, 24, $1305-1315$.

[25] L. Haar, NBS/NRC Steam Tables (Eds.: J. S. Gallagher, G. S. Kell), Hemisphere, Washington, 1984, pp. 14-15, 165, 263.

[26] C. Yoon, M. X. Yang, G. A. Somorjai, Catal. Lett. 1997, 46, 37-41.

[27] Y. Kitayama, M. Muraoka, M. Takahashi, T. Kodama, E. Takahashi, M. Okamura, JAOCS 1997, 74, 526-529.

[28] G. W. Huber, J. W. Shabaker, S. T. Evans, J. A. Dumesic, Appl. Catal. B: Environm. 2006, 62, 226-235.

[29] A. V. Kirilin, A. V. Tokarev, E. V. Murzina, L. M. Kustov, J. Mikkola, D. Y. Murzin, ChemSusChem 2010, 3, 708-718.

[30] J. W. Shabaker, R. R. Davda, G. W. Huber, R. D. Cortright, J. A. Dumesic, J. Catal. 2003, 215, 344-352.

Received: October 29, 2010

Revised: December 2, 2010

Published online on January 27, 2011 